\title{
Cross-modal bias and perceptual fusion with auditory-visual spatial discordance
}

\author{
PAUL BERTELSON and MONIQUE RADEAU \\ Université libre de Bruxelles, B-1050, Brussels, Belgium
}

\begin{abstract}
Investigations of situations involving spatial discordance between auditory and visual data which can otherwise be attributed to a common origin have revealed two main phenomena: cross-modal bias and perceptual fusion (or ventriloquism). The focus of the present study is the relationship between these two. The question asked was whether bias occurred only with fusion, as is predicted by some accounts of reactions to discordance, among them those based on cue substitution. The approach consisted of having subjects, on each trial, both point to signals in one modality in the presence of conflicting signals in the other modality and produce same-different origin judgments. To avoid the confounding of immediate effects with cumulative adaptation, which was allowed in most previous studies, the direction and amplitude of discordance was varied randomly from trial to trial. Experiment 1, which was a pilot study, showed that both visual bias of auditory localization and auditory bias of visual localization can be observed under such conditions. Experiment 2, which addressed the main question, used a method which controls for the selection involved in separating fusion from no-fusion trials and showed that the attraction of auditory localization by conflicting visual inputs occurs even when fusion is not reported. This result is inconsistent with purely postperceptual views of cross-modal interactions. The question could not be answered for auditory bias of visual localization, which, although significant, was very small in Experiment 1 and fell below significance under the conditions of Experiment 2.
\end{abstract}

Studies of reactions of human subjects to situations involving spatial discordance between auditory and visual data which can otherwise be related to a common distal event have revealed interesting interactions, showing that such data are not processed as independently as the notion of separate sense modalities seems to imply.

Interactions have been observed at two different levels: (1) immediate reactions, or reactions to the discordant data; and (2) aftereffects of exposure to discordant data on reactions to unimodal data.

The present study was concerned with immediate reactions. Investigations of such reactions have resorted to two main classes of tasks, discordance detection and unimodal localization.

In discordance-detection experiments, subjects are presented with discordant data and are instructed to judge whether these data come from the same or from different locations (Choe, Welch, Gilford, \& Juola, 1975; Klemm, 1909; Witkin, Wapner, \& Leventhal, 1952), or, in a slightly different version,

This research was supported by the Belgian Fonds de la Recherche Fondamentale Collective under Contract 2.4505 .80 with $P$. Bertelson and M. Wajskop. M. Radeau is Chercheur Qualifié of the Belgian Fonds National de la Recherche Scientifique. Thanks are extended to J. M. Faverge for help in developing the method for calculating corrected baselines and to P. Viviani for fruitful discussions. Reprint requests may be directed to: P. Bertelson, Laboratoire de Psychologie expérimentale, 117, avenue Ad. Buyl, 1050 Bruxelles, Belgium. whether they experience perceptual fusion (Jack \& Thurlow, 1973; Radeau \& Bertelson, 1977; Thurlow \& Jack, 1973). A basically similar task is the one in which the subject is asked to match auditory data with one of several possible visual origins (Jackson, 1953). The finding of interest here is that, under some conditions, subjects fail to detect discordance, or report fusion, in the presence of discordances that are easily detected when occurring within one modality, or across modalities in other conditions. Such perceptual fusion has sometimes been called ventriloquism, since it seems to be the basis for the illusion created by the ventriloquist that the speech he produces without visible facial movements comes from the mouth of his puppet.

In unimodal localization experiments, subjects are instructed to indicate the origin of data reaching one modality, the target modality, in the presence of spatially discordant data in the other modality, the competing modality, which he is supposed to ignore. Pointing has been the response in the majority of studies (Bermant \& Welch, 1975; Pick, Warren, \& Hay, 1969; Radeau, 1974; Radeau \& Bertelson, 1976; Warren, 1979; Weerts \& Thurlow, 1971), but proximity judgments have also been used (Klemm, 1909; Thomas, 1941; Radeau \& Bertelson, Note 1). The finding of interest here is cross-modal bias: responses are displaced in the direction of the competing data, in spite of instructions to ignore them. Substantial biasing effects of visual data on auditory localization 
have been found in all the studies. Regarding the opposite effect, auditory bias of visual localization, which in some studies has not been looked for (Bermant \& Welch, 1975; Thomas, 1941; Weerts \& Thurlow, 1971), the evidence is somewhat ambiguous: it showed up in the studies by Klemm (1909) and Radeau and Bertelson (1976) but not in those by Pick et al. (1969) and Warren (1979).

All previous investigations have resorted to either one or the other of the two types of tasks. As a result, little is known about the relation between perceptual fusion and cross-modal bias. In the present study, an attempt was made to explore the problem by having subjects give same-different origin judgments and unimodal pointing responses on the same trials.

Examination of the procedure used in previous studies, however, revealed a methodological problem which had to be solved first: How could cross-modal bias be measured? Most studies of bias have involved long series, generally whole sessions, with a constant discordance (Bermant \& Welch, 1975; Pick et al., 1969; Warren, 1979, Experiments 1 and 2; Weerts \& Thurlow, 1971). The usual measure of bias is based on the difference between mean localization error during a series of trials with conflicting inputs and during an initial series of unimodal trials with target data only. The obtained measure, which can be called cumulative bias, confounds the immediate biasing effect of the conflicting input with the cumulative adaptation resulting from previous trials. The immediate effect, or immediate bias, can at each moment be measured by the difference between error in localizing the target stimulus accompanied by a conflicting stimulus and error in localizing the target stimulus alone. This value, however, will normally change with adaptation, since cumulative bias goes to an asymptote (Radeau \& Bertelson, 1976; Weerts \& Thurlow, 1971). For that reason, immediate bias should be measured in a condition minimizing the occurrence of adaptation, that is, with the discordance changing in direction from trial to trial. Until the recent study by Warren (1979, Experiment 3), which was published after the present experiments had been completed, the experiment by Thomas was the only one in which this condition was clearly met (Klemm's description of his procedure was not sufficiently detailed to establish how he organized the order of his trials).

The purpose of Experiment 1 was to determine if cross-modal bias could be observed when contamination from adaptation was controlled for. The subjects were presented with trains of synchronous point flashes of light and sound pulses, coming from sources with azimuthal separation that were varied randomly in direction and in magnitude from trial to trial. The subjects were instructed, in one session, to point with the hidden hand to the direction of the light and, in another session, to the direction of the sound.

\section{EXPERIMENT 1}

\section{Method}

Apparatus. The apparatus was a slightly modified version of the one used by Radeau and Bertelson (1976). Basically, it consisted of a semicylindrical enclosure divided into two compartments by a horizontal panel. The wall of the upper compartment was opaque. A mobile display holder, attached to a vertical rod that could be moved around the outer side of the cylinder by a pivot system, protruded under the lower edge of the screen through a light trap. This display holder held a small loudspeaker, $4 \mathrm{~cm}$ above the horizontal panel, and a neon pea lamp, $3 \mathrm{~cm}$ above the panel. For the present experiment, two other display sets, each consisting of a loudspeaker and a lamp vertically aligned with the corresponding elements on the mobile holder, were attached to the opaque screen at $10 \mathrm{deg}$ to each side of the median plane. The three loudspeakers were Philips LBC 1013/00 earphones (3-mm diam), through which sound bursts produced by 50 -msec pulses of $300 \mathrm{~Hz}$ ac were presented. The lamps were red LED lamps (Monsanto Type MV 50). The lower compartment was limited by a transparent screen. To point, the subject placed the tip of the finger against the transparent screen. The experimenter, who sat on the other side of the apparatus, could read the position of the finger to the nearest degree on a graduation painted on the screen. A pointer fastened to the movable target came into contact with the same graduation; this could also be used by the experimenter to position the target.

The subject sat on an adjustable stool and placed his chin on a chinrest, which was positioned at the edge of the horizontal panel, in the axis of the cylinder. The apparatus was designed to maintain the subject in complete darkness and to provide him with no visual stimulation other than that provided by the display lamp in use.

Procedure. Each of the 16 subjects served in one visual and one auditory session, during which all pointing had to be based on signals in the corresponding modality. In each session, there were conflict trials and control trials. On control trials, only visual or auditory signals were produced, depending on the type of session. On conflict trials, signals in both modalities were produced in exact synchrony. The signals always consisted of a train of six 50 -msec pulses separated by 450 -msec intervals. For conflict trials, the signal to be pointed at was called the target signal, and the other signal was called the competing signal.

Signals of control trials and target signals of conflict trials were all made to appear either $10 \mathrm{deg}$ left or $10 \mathrm{deg}$ right of the median plane (i.e., they were delivered by one of the fixed display units). Competing signals of conflict trials were delivered by the mobile display unit 7,15 , or $25 \mathrm{deg}$ left or right of the target signal. There were, thus, 12 different types of conflict trials, depending on the position of the target signal and on the size and direction of the azimuthal separation between target and competing signal. One session involved 12 successive blocks of 14 trials each, that is, one conflict trial of each type and two control trials, one with the signal at 10 deg left and one with the signal at 10 deg right. A different order of the 14 trials was used for each block.

Trials followed each other at 11 -sec intervals, and there was a 5-min pause in the middle of the session.

The instructions were to indicate the origin of the target signal by placing the tip of the right index finger (all subjects were righthanded) against the transparent screen immediately under the source of that signal and to ignore the competing signal. The subjects were told that the two signals might come from the same place on some trials and from different places on other trials. The subjects were also asked to bring the right hand back to a resting position on top of the left hand between trials. The left hand rested on the tabie, grasping the holder of the chinrest. On control trials, the subjects were instructed to point at the signal and also to report verbally the absence of the competing signal. Control trials thus also functioned as catch trials, forcing attention to the competing signal.' 
Half of the subjects were given the visual session first, and the other half, the auditory session first.

Subjects. Sixteen right-handed university students volunteered to serve as subjects for about 100 Belgian francs per session.

\section{Results}

For each subject, each session, and each of the six types of conflict trials ( $7 \mathrm{deg}$ left, $7 \mathrm{deg}$ right, $15 \mathrm{deg}$ left, etc.), the mean shift in pointing as a result of cross-modal bias was computed by subtracting the mean pointing error on the conflict trials from the mean pointing error on control trials. The difference was scored as positive when it went in the direction of attraction by the competing signal. The mean of the two mean shifts corresponding to a same angular separation (e.g., $25 \mathrm{deg}$ left and $25 \mathrm{deg}$ right) appears in Table 1, expressed in degrees (absolute crossmodal bias) and in percent of the angular separation (percent cross-modal bias). Results of one-tailed $t$ tests applied to the hypothesis of null bias are also given.

The visual bias of auditory localization is substantial. It increases in absolute value with angular separation, although less than proportionately. The auditory bias of visual localization is much smaller, but nevertheless reaches significance $(p<.0005)$ at all separations.

\section{Discussion}

A positive answer can be given to the methodological question that has been asked: Both visual bias of auditory localization and auditory bias of visual localization can be observed in a condition in which they cannot be attributed to cumulative adaptation. Further work on bias should preferably be done with the kind of variable discordance procedure that has been used.

The ambiguity of the evidence regarding auditory bias of visual localization was mentioned in the introduction. One reason the effect, which was observed by neither Pick et al. (1979) nor Warren (1979), showed up in the present study as well as in Radeau \& Bertelson (1976, Experiment 1) might be because, in both studies, our subjects always had to monitor the competing auditory signals, in order to be able to signal their absence on control catch trials, whereas the

Table 1

Experiment 1: Absolute (in Degrees) and Relative (in Percent) Cross-Modal Biases as Functions of Angular Separation

\begin{tabular}{ccccc}
\hline \multirow{2}{*}{$\begin{array}{c}\text { Separation } \\
\text { (in Degrees) }\end{array}$} & \multicolumn{2}{c}{$V(\mathrm{~A})$} & \multicolumn{2}{c}{$\mathrm{A}(\mathrm{V})$} \\
\cline { 2 - 5 } & Degrees & Percent & Degrees & Percent \\
\hline 7 & $3.99^{*}$ & 57.0 & $.34^{*}$ & 4.8 \\
15 & $6.27^{*}$ & 41.8 & $.47^{*}$ & 3.1 \\
25 & $8.16^{*}$ & 32.6 & $.61^{*}$ & 2.4 \\
\hline
\end{tabular}

Note-V $(A)=$ visual bias of auditory localization; $A(V)=a u d i$ tory bias of visual localization. ${ }^{*} p<.0005$ by one-tailed $t$ test. subjects in the other studies could ignore them. However, this factor cannot explain why the effect was so much smaller in the present study than in our previous one, in which, in the Experiment 1 condition without visual texture, it reached $1.66 \mathrm{deg}$ for the means of the first five trials and $1.56 \mathrm{deg}$ for the very first trial of each subject.

\section{EXPERIMENT 2}

Having established the reality of cross-modal bias, we can proceed to examine its relation to the phenomenon of perceptual fusion. Although it has generally been assumed that the two phenomena are somehow related, except for some suggestions developed by Choe et al. (1975) and discussed by Bertelson and Radeau (1976), ideas regarding the relationship have mostly remained implicit.

Perceptual fusion can be viewed, as proposed by Choe et al., as the outcome of a statistical decision process. Information about spatial location available in each modality gives rise to two registered locations. Fusion occurs when registered locations are closer together than some criterion value. In such a model, factors which are known to influence fusion, such as synchronism and realistic context (Jack \& Thurlow, 1973; Radeau \& Bertelson, 1977; Thurlow \& Jack, 1973), can work (1) by bringing registered locations closer together (this hypothetical phenomenon will be called attraction), and (2) by affecting the setting of the criterion. There is, of course, no a priori reason to eliminate the possibility of an influence at both levels. Choe et al. produced data which they thought were inconsistent with the attraction notion, but Bertelson \& Radeau (1976) showed that the particular attraction mechanism that was eliminated by their application of detection theory was an implausible one, which nobody would have wanted to defend. Our conclusion was that the issue was an open one.

The occurrence of cross-modal bias would at first sight support the attraction notion: It seems reasonable to assume that the locations subjects point to correspond to the registered locations used in the fusion vs. no-fusion decision. There are, nevertheless, ways of accounting for bias which are compatible with a criterion-shift interpretation of fusion. One which has been proposed by several authors (e.g., Howard \& Templeton, 1966, p. 361; Kaufman, 1974, p. 437), including Choe et al., is that when fusion occurs, spatial data in one modality are simply ignored and the fused percept is given a location on the basis of the spatial data from the other modality. This hypothetical mechanism has been called cue substitution (Radeau \& Bertelson, 1976). Those who have proposed it have generally specifically considered substitution of visual data for auditory data, but allowing for the occasional occurrence of the in- 
verse phenomenon makes for no logical difficulty, as we have noted (Radeau \& Bertelson, 1976). Another possibility has been considered by Bertelson and Radeau (1976): The fusion vs. no-fusion decision is made on the basis of unbiased registered locations, but when, and only when, fusion results, bias occurs, producing perceived locations closer together than the original registered locations. In this "bias contingent on fusion" hypothesis, bias is thus considered as a particular manifestation of intermodal patterning, a theoretically attractive possibility.

One possible way of testing for the occurrence of attraction before fusion was to present subjects with discordant pairs of signals in the two modalities, and to have them point to signals in one modality and also give same-different origin judgments. The two last hypotheses, cue substitution and bias contingent on fusion, share the prediction that there must be no bias on trials in which separate origins are reported.

Testing that prediction, however, poses the question of which control values to use for measuring bias. The problem is that no-fusion trials are necessarily those on which the normal variability of localization mechanisms will have produced registered locations with large separations. Fortunately, it is possible to estimate the effect of this selection process by a method that will be described in the Results section.

In Experiment 2, subjects performed exactly the same unimodal pointing tasks as in Experiment 1, and, in addition, indicated on each trial whether the signals appeared to come from the same or from different directions.

\section{Method}

The apparatus and the procedure were exactly as in Experiment 1 , except for the addition of an instruction to report, on each conflict trial, whether the two signals came from the same or from different directions. The subjects were not required to give the two responses in a particular order. Eight new subjects from the same population were tested for two sessions each.

\section{Results}

The overall mean biases, irrespective of samedifferent-origin judgment, calculated exactly as in
Table 2

Experiment 2: Absolute (in Degrees) and Relative (in Percent) Cross-Modal Biases as Functions of Angular Separations and Percent "Same-Origin" Responses (P)

\begin{tabular}{cccccccc} 
& \multicolumn{3}{c}{$\mathrm{V}(\mathrm{A})$} & & \multicolumn{2}{c}{$\mathrm{A}(\mathrm{V})$} \\
\cline { 2 - 4 } \cline { 6 - 7 } $\begin{array}{c}\text { Separa- } \\
\text { tion (in }\end{array}$ & \multicolumn{2}{c}{ Bias } & & \multicolumn{2}{c}{ Bias } \\
Degrees) & $\mathrm{P}$ & Degrees & Percent & & P & \multicolumn{2}{c}{ Degrees Percent } \\
\hline 7 & 79 & $3.39^{* *}$ & 48.4 & 74 & .19 & 2.7 \\
15 & 48 & $4.36^{*}$ & 29.1 & 38 & .31 & 2.1 \\
25 & 12 & $3.88^{*}$ & 15.5 & & 21 & .13 & .5 \\
\hline
\end{tabular}

${ }^{*} p<.005$ by one-tailed $t$ test.

$* * p<.0005$ by one-tailed t test.

Experiment 1, are given in Table 2. The visual bias of auditory localization is significant at all separations. Contrary to the results of Experiment 1, however, it does not increase in absolute value with separation. In an ANOVA carried out on individual V(A) values from the two experiments, the Experiments by Angular Separation interaction was significant $[F(2,44)=3.88$, $\mathrm{p}=.05]$. Another difference between the present results and those of Experiment 1 concerns auditory bias of visual localization, which does not reach a significance at any separation. In an ANOVA carried out on $A(V)$ values from the two experiments, the Experiments effect falls just short of the .05 significance level $[F(1,22)=3.38$; for $p=.05, F(1,22)=$ 4.30].

The percentages of "same-origin" judgments are also given in Table 2. ANOVA revealed no systematic difference between the two pointing instructions $[F(1,7)<1]$ or any interaction between that source of variation and angular separation $[F(2,14)=$ 2.09].

The data of direct interest for the question of the relation between bias and fusion appear in Table 3 , in which pointing errors have been analyzed as a function of the type of response given in the samedifferent origin task, in the following way.

For each subject, each session, each of the six discordances ( 3 angles by 2 directions), and each location of the target (10 deg left or $10 \mathrm{deg}$ right), the

Table 3

Experiment 3: Uncorrected and Corrected Cross-Modal Biases (in Degrees) as Functions of Same-Different Origin Judgment

\begin{tabular}{|c|c|c|c|c|c|c|c|}
\hline \multirow[b]{2}{*}{$\begin{array}{c}\text { Separation } \\
\text { (in Degrees) }\end{array}$} & \multirow[b]{2}{*}{$\begin{array}{l}\text { Percent } \\
\text { Fusion }\end{array}$} & \multicolumn{3}{|c|}{ Fusion Trials } & \multicolumn{3}{|c|}{ No-Fusion Trials } \\
\hline & & $\begin{array}{c}\text { Uncorrected } \\
\text { Bias }\end{array}$ & $\begin{array}{c}\text { Corrected } \\
\text { Baseline }\end{array}$ & $\begin{array}{l}\text { Corrected } \\
\text { Bias }\end{array}$ & $\begin{array}{c}\text { Uncorrected } \\
\text { Bias }\end{array}$ & $\begin{array}{c}\text { Corrected } \\
\text { Baseline }\end{array}$ & $\begin{array}{c}\text { Corrected } \\
\text { Bias }\end{array}$ \\
\hline \multicolumn{8}{|c|}{ Auditory Target Modality } \\
\hline $\begin{array}{r}7 \\
15 \\
25\end{array}$ & $\begin{array}{l}79 \\
48 \\
12\end{array}$ & $\begin{array}{r}3.66 \\
8.15 \\
14.81\end{array}$ & $\begin{array}{l}+.64 \\
+2.27 \\
+4.20\end{array}$ & $\begin{array}{r}3.02 * \\
5.88 \dagger \\
10.61 \dagger\end{array}$ & $\begin{array}{r}-.69 \\
1.07 \\
2.75\end{array}$ & $\begin{array}{l}-3.94 \\
-2.64 \\
-.45\end{array}$ & $\begin{array}{l}3.15^{*} \\
3.00^{* *} \\
3.20^{* *}\end{array}$ \\
\hline \multicolumn{8}{|c|}{ Visual Target Modality } \\
\hline $\begin{array}{r}7 \\
15 \\
25\end{array}$ & $\begin{array}{l}74 \\
38 \\
21\end{array}$ & $\begin{array}{r}.55 \\
1.05 \\
2.42\end{array}$ & $\begin{array}{l}+.17 \\
+.69 \\
+1.00\end{array}$ & $\begin{array}{r}.32 \\
.36 \\
1.42\end{array}$ & $\begin{array}{l}-1.02 \\
-.25 \\
-.38\end{array}$ & $\begin{array}{l}-.85 \\
-.32 \\
-.25\end{array}$ & $\begin{array}{r}-.17 \\
.07 \\
-.13\end{array}$ \\
\hline
\end{tabular}

Note-Values in italics are based on incomplete data. All $p$ values are based on one-tailed $t$ tests.

${ }^{*} p<.01 . \quad * * p<.005 . \quad t_{p}<.0005$. 


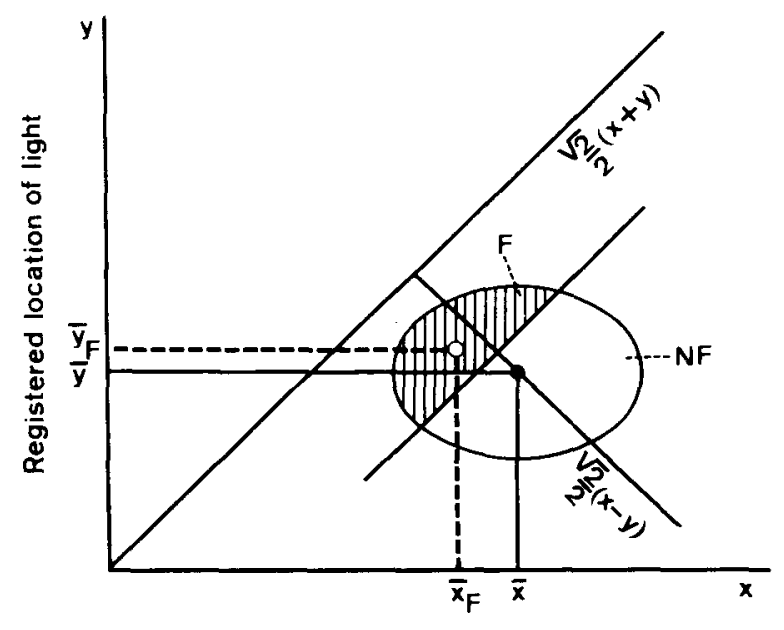

Registered location of sound

Figure 1. Method for estimating the effect of selection, in the absence of attraction, on mean registered locations of sound and light, separately for fusion and no-fusion trials: case of $\bar{x}>\bar{y}$.

mean pointing error was calculated separately for fusion and for no-fusion trials. Two subjects gave no "same-origin" responses at $25 \mathrm{deg}$ separation under either pointing instruction, so the data for those conditions are incomplete. The difference between mean pointing errors on conflict trials and mean pointing errors on control trials with the same target location gives the "uncorrected biases" whose means over subjects, discordance directions, and target locations are given in Table 3. As usual, biases are counted as positive when they go in the direction of attraction by the competing signal. Considerable visual biases of auditory pointing are observed on fusion trials, but not on no-fusion trials, where the value obtained reaches significance at 25 deg separation only.

However, as noted in the introduction, by using mean pointing errors on control trials as baselines to measure bias, one allows the result to be influenced by the selection involved in separating fusion from no-fusion trials. This inconvenience can be remedied by using, as baselines, the mean pointing errors that would occur, respectively, on fusion and on no-fusion trials under the hypothesis that fusion results from a statistical decision on the basis of unbiased registered locations. The method is explained in the Appendix and illustrated in Figure 1. Each subject's distribution of pointing errors on corresponding control trials is used to represent registered locations of a particular target at a particular location.

The principle of the method is that, on each trial, one pair, $x, y$, of registered locations is obtained through sampling from the corresponding distributions. Fusion occurs when the difference $(x-y)$ is smaller than a criterion value. In Figure 1, this corresponds to partitioning the two-dimensional distribution along the diagonal at a position along the $(x-y)$ axis, which gives the observed value of $\mathrm{p}$, proportion of same-origin responses. If one assumes that $x$ and $y$ are both normally distributed, the mean $x$ and $y$ values of each of the two truncated distributions can be obtained for any proportion of fusion trials, using the table of the normal curve.

Following the preceding procedure, corrected baselines were obtained separately for each subject, pointing instruction, discordance, and target location. These corrected baselines were used for the calculation of corrected biases. Both measures, averaged over subjects, discordance directions, and target locations, appear in Table 3.

At the level of auditory pointing, corrected biases are significant at each separation for both no-fusion and fusion trials. For visual pointing, no corrected bias reaches significance. An ANOVA was applied to individual corrected biases on fusion and nofusion trials. For $A(V)$, the type of trial (fusion vs. no-fusion) effect is not significant $[F(1,7)=2.26]$. For V(A), the Type of Trial by Angular Separation interaction is significant $[\mathrm{F}(2,11)=15.1, \mathrm{p}<.005]$. This effect corresponds to the fact that V(A) does not increase with separation on no-fusion trials, but does increase markedly on fusion trials.

\section{GENERAL DISCUSSION}

(1) The main outcome of Experiment 1 is that attraction of auditory registered locations by synchronous visual signals occurs even on those trials in which no perceptual fusion is reported. Neither cue substitutions nor bias contingent on fusion offer sufficient explanations for cross-modal bias.

(2) On the other hand, corrected biases are not exactly equal on fusion and no-fusion trials: They are larger on fusion trials at the larger separations. The existence of such an interaction suggests that a second cause of attraction might operate on fusion trials. Therefore, the possibility must be considered that, besides the attraction operating on all trials, another mechanism, one which might obey the cue substitution principle, might work on some of the fusion trials.

(3) Regarding the debate triggered by Choe et al.'s (1976) paper, the proposal that auditory-visual interactions can be accounted for without recourse to any perceptual effect, solely on the basis of criterion shifts, becomes clearly untenable. This, of course, must not be taken as implying that fusion is not influenced by criterion-setting processes. As noted earlier (Bertelson \& Radeau, 1976), only such effects can explain the extreme cases of ventriloquism that have been reported by participants in visual and auditory inversion experiments (Stratton, 1897; Young, 1928).

(4) With auditory-visual discordance (but not with visuoproprioception: see Pick et al., 1969, and Warren, 
1979), total bias is typically less than the experimental discordance. As Warren (1979) notes, this means that, given a pair of signals, the subject does not indicate the same location when under auditory and under visual pointing instructions. It is tempting to ask if this result would hold when fusion trials only are considered. A positive answer would imply that the cross-modal event resulting from fusion does not have a single location, but can be located in different places according to pointing instructions. The idea that localization processes are affected by task demands has already been formulated to account for the demonstrated influence of pointing instructions on adaptation to cross-modal discordance. Both Canon (1970) and Radeau (1974) found that the aftereffects of exposure to auditory-visual discordance obtained, respectively, in visual and auditory localization depended on which modality served as pointing target during exposure. The corresponding effect was later obtained in the case of haptic-visual conflict by Kelso, Cook, Olson, and Epstein (1975).

The question can be approached indirectly through the data of Experiment 2. If, for each separation, we add the two mean uncorrected biases for fusion trials (Table 3), we obtain values that fall short of the corresponding angular separations: $4.21 \mathrm{deg}$ for $7 \mathrm{deg}$ separation, $9.20 \mathrm{deg}$ for $15 \mathrm{deg}$, and $15.23 \mathrm{deg}$ for $25 \mathrm{deg}$. This result is favorable to the variable location interpretation. The support is called indirect, however, because the method is based on two assumptions which, although reasonable, lack formal demonstration: (1) that the same origin judgment occurs on comparable trials on the two sessions with opposite pointing instructions, or, in other words, that had auditory pointing instructions been given on the fusion trials of the visual pointing session, the same distribution of pointing errors as on the corresponding trials of the auditory pointing session would have been obtained; and (2) that the amount of bias is not strongly influenced by the location of the target stimulus, since our total biases were calculated using component values that were all measured with the target stimuli at the same two 10-deg-left and 10-degright locations.

(5) Pointing responses were affected by the introduction, in Experiment 2, of the same-different origin task: The auditory bias of visual pointing ceased to be significant and the visual bias of auditory localization ceased to increase with separation, which meant an important reduction of bias at the larger separations. There is no ready explanation for these particular effects. However, as suggested in the introduction, they add to other data which suggests that unimodal localization is under the influence of a number of so far insufficiently elucidated factors. At the methodological level, the lack of invariance of the bias measures limits the possible uses of the dual task procedure.
(6) In Experiment 2, the percentage of "same origin" responses was not affected by type of pointing instruction. This result diverges from one obtained repeatedly in previous studies (Radeau, 1974; Radeau \& Bertelson, 1976): When, at the completion of several sessions of unimodal pointing with a $15 \mathrm{deg}$ discordance, subjects were asked whether the auditory and visual signals had generally been coming from the same place, most of those who had been working under auditory pointing instructions reported noticing the discrepancy, while almost all of those who had been working under visual pointing instructions failed to report it. The fact that this difference was not observed in the present situation suggests that it only shows up when the subject has no warning that he will be questioned about the respective origins of the two signals and has to rely on incidental memories. When he is warned beforehand, he can judge discordances with equal efficiency, whatever the pointing instructions. This finding does not conflict with our previous interpretation in terms of a greater propensity to ignore the spatial origin of auditory data than that of visual data unless the task forces attention to it.

\section{REFERENCE NOTE}

1. Radeau, M., \& Bertelson, P. Auditory-visual intermodal bias and temporal relations between signals. Manuscript in preparation.

\section{REFERENCES}

Bermant, R. I., \& WELch, R. B. The effect of degree of visualauditory stimulus separation and eye position upon the spatial interaction of vision and audition. Perceptual and Motor Skills, $1976,43,487-493$.

Bertelson, P., \& Radeau, M. Ventriloquism, sensory interaction, and response bias: Remarks on the paper by Choe, Welch, Gilford, and Juola. Perception \& Psychophysics, 1976, 19, 531-535.

CANON, L. K. Intermodality inconsistency of input and directed attention as determinants of the nature of adaptation. Journal of Experimental Psychology, 1970, 84, 141-147.

Choе, C. S., Welch, R. B., Gilford, R. M., \& Juola, J. F. The "ventriloquist effect": Visual dominance or response bias? Perception \& Psychophysics, 1975, 18, 55-60.

Faverge, J. M. Méthodes statistiques en psychologie appliquée (Vol. 2). Paris: Presses Universitaires de France, 1972.

Howard, I. P., \& Tem Pleton, W. B. Human spatial orientation. New York: Wiley, 1966.

JACK, C. E., \& ThuRLow, W. R. Effects of degree of visual association and angle of displacement on the "ventriloquism" effect. Perceptual and Motor Skills, 1973, 37, 967-979.

JACKson, C. V. Visual factors in auditory localization. Quarterly Journal of Experimental Psychology, 1953, 5, 52-65.

KaUfman, L. Sight and mind. New York: Oxford University Press, 1974.

Kelso, J. A. S., Cook, E., Olson, M. E., \& Epstein, W. Allocation of attention and the locus of adaptation to displaced vision. Journal of Experimental Psychology: Human Perception and Performance, 1975, 1, 237-245.

KLEMm, O. Lokalisation von Sinneseindrücken bei disparaten Nebenreizen. Psychologische Studien (Wundt), 1909, 5, 73-161.

Pick, H. L., JR., Warken, D. H., \& HaY, J. C. Sensory con- 
flict in judgments of spatial direction. Perception \& Psychophysics, 1969, 6, 203-205.

RADEAU, M. Adaptation au déplacement prismatique sur la base d'une discordance entre la vision et l'audition. L'Année Psychologique, 1974, 74, 23-34.

Radeau, M., \& Bertelson, P. The effect of a textured visual field on modality dominance in a ventriloquism situation. Perception \& Psychophysics, 1976, 20, 227-235.

Radenu, M., \& Bertelson, P. Adaptation to auditory-visual discordance and ventriloquism in semirealistic situations. Perception \& Psychophysics, 1977, 22, 137-146.

Stratton, G. M. Vision without inversion of the retinal image. Psychological Review, 1897, 4, 341-360, 463-481.

Tном⿰s, G. J. Experimental study of the influence of vision on sound localization. Journal of Experimental Psychology, 1941, 28, 167-177.

ThurLow, W. R., \& JAck, C. E. Certain determinants of the "ventriloquism effect." Perception and Motor Skills, 1973, 36, 1171-1184.

WARREN, D. H. Spatial localization under conflict conditions: Is there a single explanation? Perception, 1979, 8, 323-337.

Weerts, T. C., Thurlow, W. R. The effect of eye position and expectation on sound localization. Perception \& Psychophysics, 1971, 9, 35-39.

Witkin, H. A., Wapner, S., \& Leventhal, T. Sound localization with conflicting visual and auditory cues. Journal of Experimental Psychology, 1952, 43, 58-67.

Youna, P. T. Auditory localization with acoustical transposition of the ears. Journal of Experimental Psychology, 1928, 11, $399-429$.

\section{NOTE}

1. The subjects were thus instructed to ignore the competing signal when pointing while at the same time paying sufficient attention to it to be able to report its absence on conflict trials. It has been objected that these instructions are equivocal. We do not think they are, from the logical point of view, since what the subjects are asked to ignore is the location of the competing signal and what they are asked to monitor is its occurrence. We agree, on the other hand, that the magnitude of cross-modal bias can very likely be influenced by the monitoring instruction.

\section{APPENDIX}

\section{Method Used for the Calculation of Corrected Baselines for Bias}

The problem is to know the mean visual registered location and the mean auditory registered location, respectively, for fusion and for no-fusion trials, given the total distributions of auditory and visual registered locations and the proportion of "fusion trials."

$$
\begin{aligned}
& x=\begin{array}{l}
\text { registered location of sound, } \\
\text { with mean } \bar{x} \text { and variance } \sigma_{x}^{2}
\end{array} \\
& y=\begin{array}{l}
\text { registered location of light, } \\
\text { with mean } \bar{y} \text { and variance } \sigma_{y}^{2}
\end{array} \\
& p=\text { proportion of fusion trials. }
\end{aligned}
$$

In the present application, the distributions of pointing errors of the subject on control trials of, respectively, auditory pointing and visual pointing sessions were used to represent the distributions of registered locations relative to the objective locations of the target signals. This procedure of course leads to an overestimation of the variances of registered locations, since pointing errors are influenced not only by the variability of target registration, but also by that of the effector system.

The distributions of $x$ and $y$ are assumed to be normal. Under the hypothesis that no attraction occurs, at least prior to fusion, the two registered locations of each conflict trial are obtained by sampling independently one value from the $x$ distribution and one value from the $y$ distribution, which gives the two-dimensional distribution represented in Figure 1 by the equal-density ellipse.

Fusion results from a statistical decision based on the magnitude of the distance $(x-y)$ : It occurs when the distance is less than a fixed criterion. This amounts to partitioning the plane $x, y$ along a parallel to the diagonal, located so that the proportion of trials lying above it is $p$. We want to know $\bar{x}_{F}$ and $\bar{y}_{F}$, the mean $x$ and $y$ for trials above the partition line, and $\bar{x}_{N F}$ and $\bar{y}_{N F}$, the mean $x$ and $y$ for trials below that line.

We start by rotating the coordinate axes by $45 \mathrm{deg}$. The new coordinates are:

$$
u=\frac{\sqrt{2}}{2}(x-y) \quad v=\frac{\sqrt{2}}{2}(x+y) .
$$

Their common variance is

$$
\sigma_{u}^{2}=\sigma_{v}^{2}=\frac{\sigma_{x}^{2}+\sigma_{y}^{2}}{2},
$$

and their product-moment correlation coefficient is

$$
r_{u k}=\frac{\sigma_{x}^{2}-\sigma_{y}^{2}}{o_{x}^{2}+\sigma_{y}^{2}} .
$$

Fusion occurs on trials with $u<u_{c}$ (criterion value). The mean $\bar{u}_{F}$ of $u<u_{c}$ is given by the formula

$$
\frac{\bar{u}_{F}-\bar{u}}{o_{u}}=\frac{f}{p}
$$

(adapted from Faverge, 1972, p. 170), where $f=$ ordinate of normal density function corresponding to cumulative density $\mathrm{p}$.

Similarly,

$$
\frac{\bar{v}_{\mathbf{F}}-\bar{v}}{o_{v}}=r_{u v} \frac{f}{p}
$$

Simple algebraic derivations give the projections of point $\bar{u}_{F}, \bar{v}_{F}$ in the original $x, y$ coordinates:

$$
\begin{aligned}
& \bar{x}_{F}=\bar{x}-\frac{f}{p} \cdot \frac{\sigma_{x}^{2}}{\sqrt{\sigma_{x}^{2}+\sigma_{y}^{2}}} \\
& \bar{y}_{F}=\bar{y}+\frac{f}{p} \cdot \frac{\sigma_{y}^{2}}{\sqrt{\sigma_{x}^{2}+\sigma_{y}^{2}}}
\end{aligned}
$$

These formulas apply for $\bar{x}>\bar{y}$; for $\bar{x}<\bar{y}$, invert the signs. $\bar{x}_{\text {NF }}$ and $\bar{y}_{\text {NF }}$ are obtained by substituting $(1-p)$ for $p$ and inverting the signs.

(Received for publication October 15, 1980; revision accepted January 20,1981 .) 